\title{
A novel role of Hippo-Yap/TAZ signaling pathway in lymphatic vascular development
}

\author{
Boksik Cha, ${ }^{1, *}$ Sungjin Moon ${ }^{2} \mathcal{E}$ Wantae Kim ${ }^{3, *}$ \\ ${ }^{1}$ Daegu Gyeongbuk Medical Innovation Foundation, Daegu 41061, ${ }^{2}$ Department of Biological Science, Kangwon National University, \\ Chuncheon 24341, ${ }^{3}$ Department of Biochemistry, Chungnam National University, Daejeon 34134, Korea
}

The lymphatic vasculature plays important role in regulating fluid homeostasis, intestinal lipid absorption, and immune surveillance in humans. Malfunction of lymphatic vasculature leads to several human diseases. Understanding the fundamental mechanism in lymphatic vascular development not only expand our knowledge, but also provide a new therapeutic insight. Recently, Hippo-YAP/TAZ signaling pathway, a key mechanism of organ size and tissue homeostasis, has emerged as a critical player that regulate lymphatic specification, sprouting, and maturation. In this review, we discuss the mechanistic regulation and pathophysiological significant of Hippo pathway in lymphatic vascular development. [BMB Reports 2021; 54(6): 285-294]

\section{INTRODUCTION}

In mammals, there exist complementary vascular networks. Blood vasculature delivers nutrients and oxygen to cells, and the lymphatic vasculature maintains fluid homeostasis by collecting and returning interstitial fluid into the bloodstream. Additionally, lymphatic vasculature regulates lipid absorption and immune response $(1,2)$. Dysfunction of lymphatic vessels is associated with several human diseases such as lymphedema, Alzheimer's disease, cancer metastasis, obesity, atherosclerosis, and inflammatory diseases (3-10). A major part of the lymphatic vascular network is established during embryonic stages (2, 11). Three stepwise events regulate the lymphatic vasculature development: specification of lymphatic endothelial cell (LECs) progenitors, differentiation of LECs and formation of lymph

*Corresponding authors. Boksik Cha, Tel: +82-53-790-5210; Fax: +82-53-790-5219; E-mail: boksik-cha@dgmif.re.kr; Wantae Kim, Tel: +82-42-821-5488; Fax: +82-42-822-7548; E-mail: wantaekim@ cnu.ac.kr

https://doi.org/10.5483/BMBRep.2021.54.6.020

Received 25 January 2021, Revised 23 February 2021, Accepted 3 March 2021

Keywords: Hippo signaling pathway, Lymphatic endothelial cells (LECs), Lymphatic vascular development, PROX1, TAZ, VEGF-C/ VEGFR3, YAP sacs, and patterning and maturation of the lymphatic vessels. Different signaling pathways such as VEGFC-VEGFR3, NOTCH, BMP, WNT, PCP, G-protein-coupled receptors (GPCRs), ECMintegrin, and mechanotransduction signaling pathway regulate LEC identity, morphology, and behaviors via their downstream kinases, adaptor molecules, and transcriptional factors (1, 12-18).

The Hippo signaling is an evolutionarily conserved organ size-control mechanism and plays pivotal roles in maintaining tissue homeostasis by regulating cell proliferation, growth, and survival (19-22). In mammals, the central transcription factors of the Hippo pathway are Yes-associated protein (YAP) and its paralogue $\mathrm{WW}$ domain-containing transcription regulator (WWTR, hereinafter referred to as TAZ) $(23,24)$. In general, the phosphorylation status has been generally accepted as the most important regulatory mechanism for determining YAP/TAZ's subcellular localization and transcriptional activity $(25,26)$. The Hippo signaling can be tightly controlled by a core kinase cascade consisting of the Ste-20 family of protein kinase MST1/2, the scaffolding protein Salvador (SAV), and large tumor suppressor kinase LATS1/2 (27-32). MOB kinase activator 1A/B (MOB1A/B) forms a complex with LATS1/2 kinases. MST1/2 activates MOB1A1B and LATS1/2 by phosphorylation. The tumor suppressor NF2/Merlin associates with LATS1/2 and accelerates LATS1/2 phosphorylation through the MST1/2-SAV complex. In parallel to MST1/2, MAP4K family kinases can also directly phosphorylate and activate $\operatorname{LATS} 1 / 2(25,33)$. The wide range of intrinsic or extrinsic signals regulate the Hippo pathway that precedes to the LATS1/2-mediated YAP/TAZ phosphorylation. As a result, YAP/TAZ are located in the cytoplasm through interaction with 14-3-3, and E3 ligase $\beta$-TrCP results in the proteasome-dependent YAP/TAZ degradation (34-37). In addition, growth factor signaling or cytoskeleton rearrangement inhibits YAP/TAZ phosphorylation by suppressing the Hippo pathway and enables YAP/TAZ to translocate into the nucleus. Then, YAP/TAZ associates with TEA domain family member 1-4 (TEAD1-4) and binds with several transcription factors SMADs, RUNXs, and p63/p73 to activate the transcriptional program involved in anti-apoptosis and cell proliferation (38, 39). Recently, several studies have identified Hippo-YAP/TAZ signaling components as novel players in lymphatic vascular development by regulating LEC specification, proliferation, and migration. Therefore, in this review, we provide the current

ISSN: 1976-670X (electronic edition)

Copyright (c) 2021 by the The Korean Society for Biochemistry and Molecular Biology

(ㄷ) This is an open-access article distributed under the terms of the Creative Commons Attribution Non-Commercial License (http://creativecommons.org/licenses/by-nc/4.0) which permits unrestricted non-commercial use, distribution, and reproduction in any medium, provided the original work is properly cited. 
findings concerning the Hippo-YAP/TAZ signaling pathway mediated regulation of lymphatic vessel formation and maturation.

\section{YAP/TAZ EXPRESSION IN LYMPHATIC VASCULATURE}

It has been reported that YAP/TAZ are dynamically expressed in blood vascular endothelial cells (BECs) during angiogenesis (40-42). Remarkably, TAZ expression has been reported to be higher in several types of BECs compared with YAP (42). YAP/ TAZ are highly restricted in the nucleus of BECs between E10.5 to 11.5. However, YAP/TAZ are also found in the cytosol of most of the brain BECs at E14.5 (42). While YAP is mainly located in the cytoplasm in the migrating tip cells, TAZ is localized in the nuclei in the retinal blood vasculature at postnatal stage P5 $(40,41)$. In addition, YAP is detectable at cellcell junctions in blood vessels of neonatal mice (43). Moreover, dynamic and differential YAP/TAZ expression patterns are observed during lymphatic vasculature development. In primary human LECs (hLECs), TAZ is expressed at a much higher level compared to YAP at the protein level. However, based on RNA-seq data, YAP expression is extremely high compared to TAZ (44). The mechanisms that regulate the post-transcriptional regulation of YAP/TAZ are still not known in LECs. TAZ is mainly located in the nuclei, but YAP is diffusely distributed in the LECs of the lymphatic plexus (45). In the newly forming lymphatic valve-endothelial cells (LV-ECs), TAZ is mainly localized in the cytoplasmic compartment at E16.5 $(44,45)$. However, TAZ is predominantly located in the nucleus of mature LV-EC after E17.5 $(44,46)$. In premature LVs CTGF and ANGPT2, the target genes of YAP/TAZ were not present, but high expression has been reported in mature LVs $(44,46)$. It has been proposed that YAP/TAZ have both distinct and redundant functions so they can compensate for each other in a context-dependent manner $(47,48)$. Single deletion of YAP or TAZ in LECs does not lead to any obvious developmental defect (44). Single deletion of TAZ leads to very mild lymphatic valve defect (45). On the contrary, genetic inactivation of both YAP/TAZ in LECs results in dramatic dilation of lymphatic vessels and structural LV deterioration $(44,45)$.

\section{UPSTREAM SIGNALS REGULATING HIPPO-YAP/TAZ PATHWAY IN LECS}

Over 20 years of research has firmly established that YAP/TAZ, the central players of the Hippo pathway, are the molecular determinants for organ size control (19). The multiple signaling pathways, such as mechanical stress, WNT, TGF- $\beta$, NOTCH, and VEGF, have been suggested to affect the growth-regulatory abilities of YAP/TAZ and interact with the Hippo pathway to coordinate numerous biological processes, indicating the significance of the signaling network (20). Here, we summarize the details of upstream signals that hold the potential to modulate the Hippo pathway in LECs (Fig. 1).

\section{VEGF-C/VEGFR3 signal}

While VEGF, a ligand of VEGFR2 is an essential factor for blood vessel development, VEGF-C is the major ligand that activates VEGFR3 for lymphatic vascular development $(1,49)$. VEGF-C/VEGFR3 interaction activates PI3K-AKT and PKC-ERK pathways to regulate LECs proliferation, survival, and migration

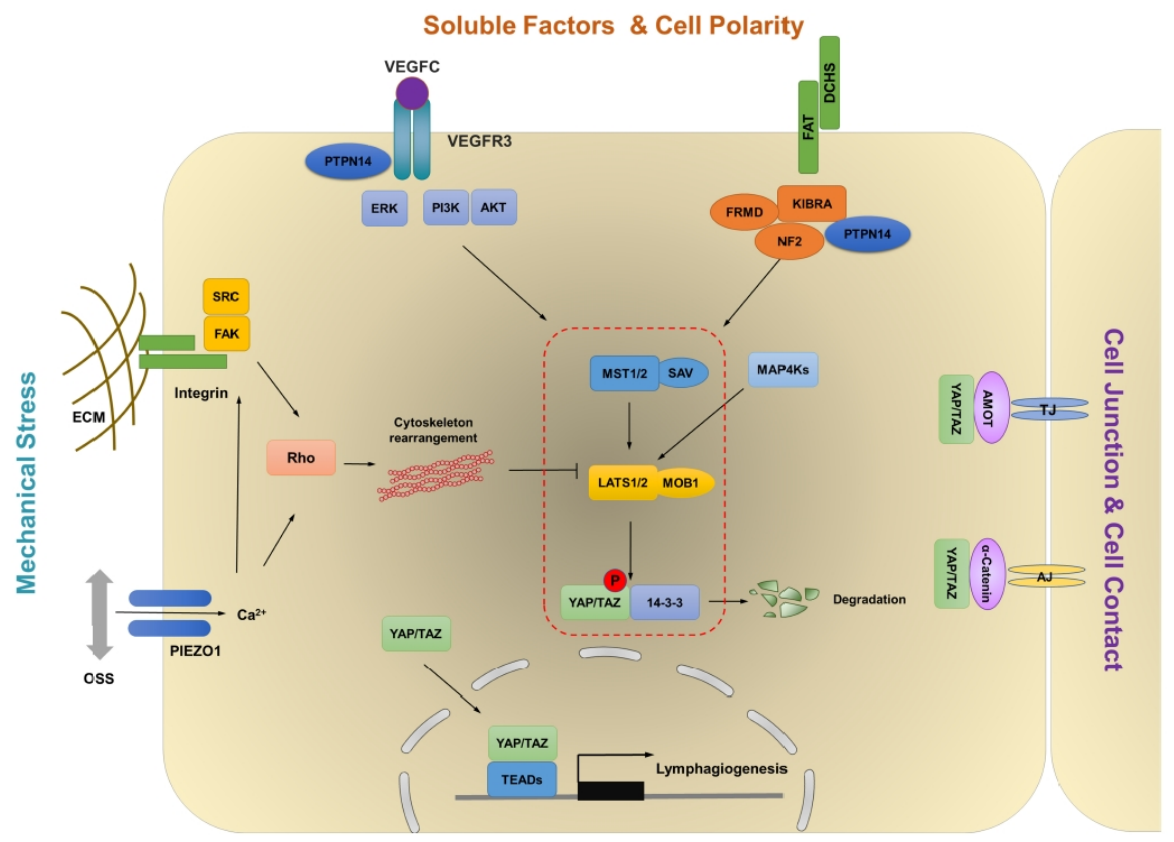

Fig. 1. Schematic diagram of the proposed model of Hippo-YAP/TAZ signaling pathway in LECs. The Hippo pathway is a kinase cascade involving MST1/2 mediated activation of LATS $1 / 2$ and LATS1/2 mediated inactivation of YAP/ TAZ through phosphorylation. Multiple stimulations including VEGF-C, mechanical stress, cell density, and cell polarity may suppress upstream kinases to activate YAP/TAZ transcriptional activity. 
(50). It has been reported that VEGF-C treatment increased phospho-LATS1 and facilitated cytoplasmic YAP whereas VEGFR3 knockdown promoted nuclear localization of YAP, thereby suggesting that VEGF-C activates the Hippo signaling to repress YAP/TAZ in hLECs (45). However, Hogan and colleagues suggested that Vegfc can promote nuclear Yap1 in a zebrafish model (51). In addition, VEGF-C decrease phospho-YAP and phospho-LATS1 in low confluent hLECs in vitro and YAP/TAZ activity is downregulated in Vegf $\mathrm{C}^{+-}$embryos (44). The activation of VEGF/VEGFR2 signaling induces PI3K-AKT and MEK-ERK signaling pathways that lead to the inhibition of MST1/2 and LATS1/2 in (52). Although Vegfc activates Yap1 in zebrafish via ERK activation, the detailed molecular mechanisms downstream of VEGF-CNEGFR3 in LECs are still unknown. Interestingly, KRAS/MAPK pathway which is triggered by VEGF-C/VEGFR3 can induce YAP expression during skin cancer progression (53). Overall, the current data suggest that YAP/TAZ are regulated by the VEGF-C/VEGFR3 signaling pathway in a high contextand cell type-specific manner.

\section{Cell polarity and cell-cell contact}

Elucidating the critical roles of apical-basal cell polarity in the regulation of the Hippo pathway provides insight to better understand the link between the cellular structural components and growth-regulatory mechanism. At the adherent junction, the FERM domain proteins Merlin (Mer) and Expanded (Ex) have been reported to connect the transmembrane proteins to the cytoskeleton. Mer and Ex genetically and functionally co-operate to mediate activation of LATS1/2 and consequent inhibition of YAP/TAZ (54). Moreover, the atypical cadherin Fat has emerged as an upstream regulator of $\mathrm{Ex}$, which promotes its junctional localization and stability (55). However, the FAT4DCHS1 signaling is essential for vertebral growth in YAP/TAZ independent manner (56). Mutations in FAT4 have been reported in Hennekam lymphangiectasia-lymphedema syndrome, features of which include lymphedema, lymphangiectasia, and mental retardation (57). Fat4 inactivation leads to dysmorphic lymphatic valves and impaired polarization of LECs in response to the flow. However, YAP/TAZ target genes are not affected by the loss of Fat4 (58). VANGL2 is also a core PCP component and YAP activity is reduced in the lung airways of Vangl $2^{\mathrm{Lp}}$ embryos (59). Looptail embryos, Vangl2 mutants, possess lymphatic valve maturation defect (13). It is still unclear whether the PCP pathway coordinates with the Hippo pathway in the lymphatic vasculature.

The Hippo-YAP/TAZ pathway regulates several cellular processes in response to cell-cell contact. Cell-adherent molecules are the regulator of the Hippo pathway. In high cell density, the Hippo pathway is activated and LATS1/2 kinase activity is increased, thereby leading to YAP/TAZ phosphorylation (36). YAP activity could be regulated by VE-cadherin-mediated cellcell contact in blood endothelial cells via PI3K-AKT $(43,60)$. We observed that YAP/TAZ activity was down-regulated in high cell density in in vitro hLECs, thereby indicating conservation of contact inhibition in LECs. During LV maturation, LV-ECs have discontinuous and low-density cell-cell junctions (13) and LV-ECs have high YAP/TAZ activity (44). Deletion of VE-cadherin from LECs leads to the up-regulation of YAP/TAZ activity in the dermal and mesenteric LECs (61). However, YAP/TAZ activity is downregulated in the intestinal LECs $(61,62)$.

Tyrosine phosphatase PTPN14 which is associated with Choanal Atresia-Lymphedema interacts with VEGFR3 and inhibits its downstream signaling cascade (63). PTPN14 also has an interesting relationship with the Hippo signaling pathway; it interacts with the Kibra and induced LATS1 activation to negatively regulate oncogenic YAP activity $(64,65)$. In addition, PTPN14 protein level has been reported to be elevated in response to an increase in cell density; the protein regulates nucleus-to-cytoplasm translocation of YAP in MCF10A cells (66).

\section{Physical signal}

Accumulating evidence has suggested YAP/TAZ as the central mechanosensor and mechanotransducer in response to several kinds of mechanical stresses including shear stress, stiffness, and cell geometry. These physical signals regulate the localization and activities of YAP/TAZ to coordinate complex organ architectures $(21,67,68)$. BECs are constantly exposed to mechanical forces generated by blood flow which affects cell proliferation and morphogenesis. Laminar shear stress (LSS) inactivates YAP/TAZ, whereas oscillatory shear stress (OSS) stimulates YAP/TAZ activity in BECs $(69,70)$. However, a following report suggested that even LSS can transiently activate YAP in BECs (71), and flow patterns could control the localization of YAP (72). Lymph flow generates shear stress in lymphatic vessels; the stress has been reported as critical for lymphatic vascular development $(14,73)$. LSS can enhance the proliferation and sprouting of LECs through ORAI1 mediated calcium influx and inhibition of NOTCH1 (73). LSS also enhances VEGF-C signaling through unknown mechanisms (74). $\mathrm{Ca}^{2+}$ entry through the ORAI channel can inhibit YAP/TAZ human glioblastoma cell lines (75). In addition, OSS is critical for lymphatic valve formation $(12,46)$. Shear stress sensing molecules such as PIEZO1 and VE-cadherin regulate lymphatic valve development $(61,62,76,77)$. PIEZO1 activation elicits transient $\mathrm{Ca}^{2+}$ influx and positively regulates nuclear localization of YAP in neural stem cells and osteoblasts (78-80). OSS increases YAP/TAZ activity and promotes nuclear localization of YAP/TAZ in in vitro cultured hLECs (46).

Physical changes induced by extracellular stiffness induce cytoskeleton rearrangement through actin remodeling, which controls the Hippo pathway in response to the activity of Integrin, Rho-GTPase, or FAK-SRC $(67,68,81,82)$. In cultured hLECs, soft matrix inhibits YAP/TAZ but promotes nuclear accumulation of GATA2 (83). In human mammary epithelial cells stretched by fluid pressure, YAP/TAZ can be activated thereby resulting in the entry of cells into the proliferative $S$ phase (84). Migrating LECs are mechanically stretched by interstitial fluid pressure thereby resulting in the swelling of the 
interstitium (85). Integrin $\beta 1$, a key component of ECM stiffness dependent YAP/TAZ activation, is necessary for inducing response to mechanical stretch to enhance VEGF-C/VEGFR3 signaling during LECs migration (85).

\section{G-protein-coupled receptors (GPCRs) signaling}

Yu and colleagues have accomplished a conceptual development in the regulation of the Hippo-YAP/TAZ pathway and G-proteincoupled receptors (GPCRs), the largest group of membrane receptors. GPCRs function as a critical upstream regulator in the Hippo pathway and relay the extracellular signal to Hippo signaling components (86). Depending on the type of ligands, GPCRs activate different types of heterotrimeric G-protein, thereby causing differential regulation of the Hippo signaling. Lysophosphatidic acid (LPA) or sphingosine 1-phosphate (S1P) promotes YAP/TAZ activation through G12/13-dependent LATS1/2 inhibition while epinephrine or glucagon suppresses YAP/TAZ activation by Gs signaling (86). Adrenomedullin (AM) and its receptor complex, the $\mathrm{G}$ protein-coupled receptor CLR (calcitonin receptor-like receptor; Calcrl) and Ramp2, play critical roles in lymphatic development during embryogenesis and maintenance of normal lymphatic function in adults $(15,87)$. LPA, a positive regulator of YAP/TAZ activity, is essential for lymphatic vascular development $(88,89)$. PROX1 and LYVE1 expression are induced by LPA stimulation in BECs (90). Also, S1P promotes lymphangiogenesis by activating S1P receptor 1 (S1PR1) which couples stringently to the Gi protein (91) and S1PR1 signaling is active in mature and quiescent lymphatic vessels during development (74). Taken together, several studies suggest that GPCRs signaling pathway regulates LEC proliferation and migration, and determines lymphatic vessel integrity and permeability. Therefore, it will be worthy to explore the potential cross-talk between GPCR and Hippo-YAP/TAZ signaling pathway.

\section{WNT signaling}

The WNT/ $\beta$-catenin signaling is a critical regulator that is involved in embryo development and tissue homeostasis. Abnormal regulation of WNT signaling causes diverse human diseases, including cancer and neurodegenerative disorders $(92,93)$. In the canonical WNT pathway, $\beta$-catenin is a major transcription factor activating WNT-responsive target gene expression. Without WNT stimulation, $\beta$-catenin is sequestered and phosphorylated by destruction complex containing Axin, APC, and GSK3 $\beta$, followed by $\beta$-TrCP-mediated proteasomal degradation in the cytosol. In response to the WNT stimulus, accumulated $\beta$-catenin translocates into the nucleus and ultimately activates WNT transcriptional program (94). Interestingly, growing evidence has suggested that WNT and Hippo pathways integrate and converge in the multiple layers of signaling pathways to respond to physiological inputs or alterations (95). Indeed, TAZ is known to functionally mediate WNT signaling (96). Also, YAP/TAZ can be seized by $\beta$-catenin destruction complex via physical interaction with Axin (97). In addition to the canonical WNT pathway, noncanonical WNT ligands regulate YAP/TAZ activation via $\mathrm{G} \alpha_{12 / 13}$-Rho-LATS signaling (98). Both canonical and non-canonical WNT signaling are critical for lymphatic vascular development $(12,99,100)$. It will be interesting to elucidate whether cross-talk between WNT-YAP/TAZ signaling is involved in this process.

\section{NOTCH signaling}

$\mathrm{NOTCH}$ signaling plays a central role in various biological processes and is activated by direct cell-cell communication between the $\mathrm{NOTCH}$ receptors and their ligands including Jagged and Delta-like. After the binding, the NOTCH receptor can be cleaved sequentially and converted into a $\mathrm{NOTCH}$ intracellular domain (NICD) that acts as a transcription factor to activate the NOTCH-responsive target gene $(101,102)$. YAP regulates expression of $\mathrm{NOTCH}$ receptors and their ligand Jagged1. In turn, NICD augments YAP/TAZ protein stability and creates a positive loop for tumor development $(103,104)$. $\mathrm{NOTCH}$ inhibits lymphatic development by repressing PROX1 expression (105). Genetic inactivation of Notch1 in LECs of mouse embryos leads to enlarge lymph sac and increase LEC populations $(16,106)$. However, inactivating the DLL4/NOTCH signaling using blocking antibodies leads to decline of lymphatic vessel density (107). Likewise, DI/4 ${ }^{+-}$mice have reduced lymphatic vascular density (74). The role of NOTCH in LECs is still not fully resolved. The molecular mechanism by which NOTCH pathway controls PROX1 is a key step that remains to be identified. Certainly, further work is necessitated to determine whether the NOTCH-NICD-YAP/TAZ positive feedback loop operates in the lymphatic vasculature.

\section{HIPPO-YAP/TAZ PATHWAY IN LYMPHATIC VASCULAR DEVELOPMENT}

\section{Role of Hippo-YAP/TAZ signaling in the specification of lymphatic endothelial cell progenitors}

Most of the embryonic LEC progenitors derive from the cardinal veins (108-110). In mouse embryos, around E9.5, a unique group of venous endothelial cells starts to express PROX1 and becomes LEC progenitors. The homeobox transcription factor PROX1 not only controls LEC cell-fate determination but also maintains their identity $(49,109,111,112)$. It has been known that COUP-TFII (111) and SOX18 (113) are required to activate PROX1 expression by binding directly to the PROX1 promoter. On the other hand, NOTCH signaling inhibits PROX1 expression during LEC cell-fate specification $(16,106)$. A Positive feedback loop between PROX1-VEGFR3 is necessary for controlling the LEC specification and for preserving LEC identity $(114,115)$ (Fig. 2). Koh and colleagues for the first time reported the presence of YAP/TAZ in the cytoplasm of most of the LEC progenitors (45). Activation of YAP/TAZ in cultured hLECs leads to down-regulation of PROX1 while knocking down of YAP/TAZ increases PROX1 expression (45). Hyperactivation of YAP/TAZ using Prox1-CreERT2; Lats $1^{\mathrm{f} / \mathrm{f}}$; Lats $2^{\mathrm{f} / \mathrm{f}}$ mouse demonstrated a reduction in the number of Prox $1^{+}$LECs in cardinal vein thereby 

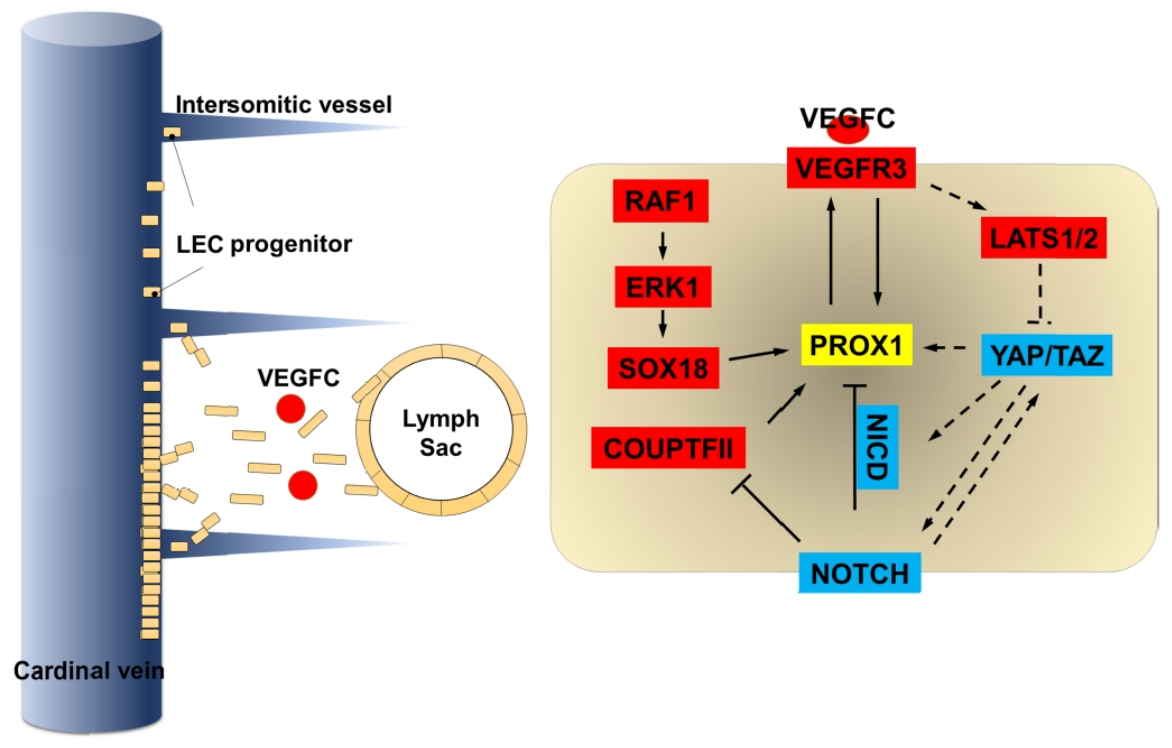

Fig. 2. Schematic representation of the molecular mechanisms controlling lymphatic specification and sprouting. PROX1 is a key transcription factor that drives lymphatic cell fate specification. Lymphatic endothelial progenitor cells expressing PROX1 arise from the cardinal veins (CV) and intersomitic veins. After specification, LECs migrate out from the CV in a VEGF-CNEGFR3 dependent manner and form lymph sac. HippoYAP/TAZ signaling regulates PROX1 expression during LEC specification and sprouting. indicating that Hippo signaling activates PROX1 expression and promotes LECs specification (45). However, Yap1 localization has been reported to be dynamically altered in parachordal LECs in zebrafish (51). Interestingly, yap1 mutants show the normal specification of lymphatic progenitors and yap1 is not necessary for specification in the zebrafish model (51).

\section{Role of Hippo-YAP/TAZ signaling in lymphatic endothelial cell migration}

In mouse embryos, around E10.5, LEC progenitors start to migrate out from the cardinal vein into mesenchyme as loosely connected spindle-shaped LECs. These LECs form lumenized lymphatic structure such as the lymph sac, the peripheral longitudinal lymphatic vessel, and the primordial thoracic duct around E11.5 $(116,117)$. Sprouting process of LECs is governed by VEGF-C/NEGFR3 in both mice and zebrafish (118-120) (Fig. 2). It appears that VEGF-CNEGFR3 signaling represses YAP/TAZ activity via LATS1 phosphorylation in vitro (45). Hyperactivation of YAP/TAZ at E10.5 reduced Prox1 expression thereby leading to a decrease in lymph sac size (45). On the other hand, yap1 mutant showed abnormal cellular sprouting in zebrafish (51). Hogan and colleagues suggested that Vegfc promotes nuclear Yap1 with subsequent regulation of LECs proliferation (51). During migration, LECs are exposed to a soft ECM environment, thereby leading to a decrease in YAP/TAZ activity and activation of GATA2-dependent VEGFR3 expression (83). However, migrating LECs are mechanically stretched because of high interstitial fluid pressure (85). Integrin $\beta 1$, a key component of ECM stiffness dependent YAP/TAZ activation, is necessary for responding to mechanical stretch to enhance VEGF-C/VEGFR-3 signaling during LECs migration (85).

\section{Role of Hippo-YAP/TAZ signaling in dermal lymphatic vascular development}

The first lymphatic vessels reach the skin from the jugular lymph sac around E12.5. Then, arising superficial lymphatic vessels on the lateral side of the embryo actively move towards until they reach the dorsal midline around E15.5-E16.5. (108, $121,122)$. They show honeycomb-like structure in the plexus region and have actively sprouting tips in the migratory front region, reflecting a dynamic process in lymphatic vascular patterning. The molecular mechanisms regulating formation of the dermal lymphatic vasculature remain incompletely understood. However, PROX1, VEGFC, FOXC2, GATA2, and NRP2 are recognized to be necessary for the dermal lymphatic development $(3,118,123-125)$. Koh and colleagues demonstrated that around E16.5, YAP/TAZ are very less expressed in the tip LECs but TAZ are nucleo-cytoplasmically located in LV-ECs in the plexus (45). Conditional deletion of YAP/TAZ in LECs from E11.5 causes enlarged, ballooned, and mispatterned lymphatic vessels with no lymphatic valves. Genetical inactivation of LATS1/2 blocks lymphatic sprouting and leads to the formation of dysmorphic lymphatic vessels (45). Lyve1-Cre; Yap ${ }^{\text {ff/f }} ; \mathrm{Taz}^{\mathrm{f} / \mathrm{f}}$ embryos have defective lymphatic vasculature with dilated vessels, fewer branch points, and migration defect around E18.5 (44). Therefore, Hippo-YAP/TAZ signaling pathway is unquestionably essential for dermal lymphatic vascular development.

\section{Role of Hippo-YAP/TAZ signaling in lymphovenous and lymphatic valve development}

Lymph returns to the blood circulatory system particularly through four lymphovenous valves (LVVs) $(126,127)$. They start forming around E12 at the junction of the jugular and subclavian veins $(128,129)$. The development of LVV starts 


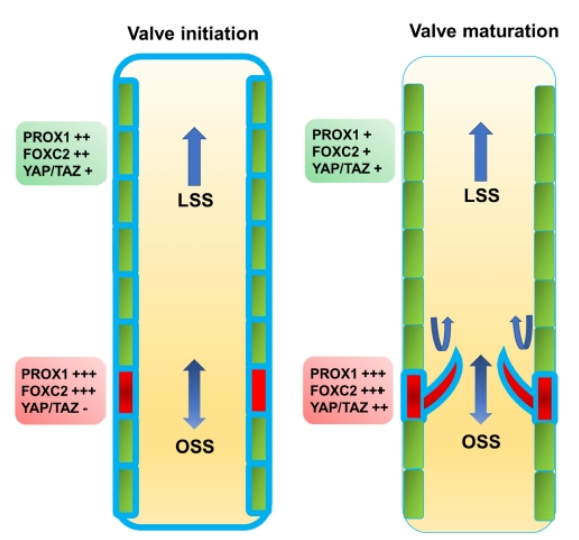

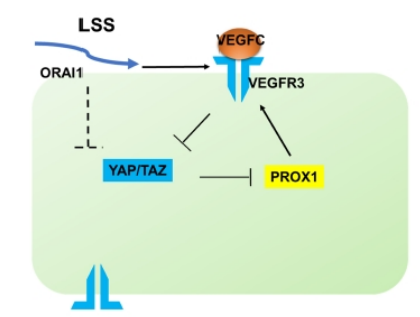

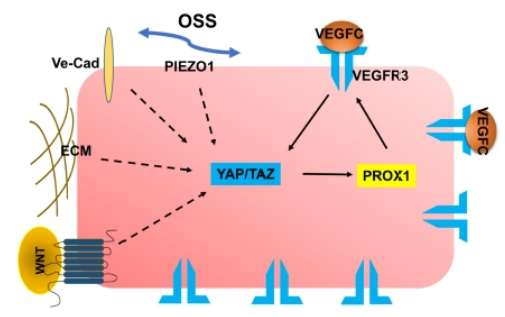

Fig. 3. A proposed mechanism of HippoYAP/TAZ signaling pathway function in lymphatic valve (LV) formation. LVs develop in a step-wise manner. LV-ECs are specified in red and LECs are in green. Blue lines indicate VEGFR3. Briefly, during the initiation stage, OSS promotes high PROX1 and FOXC2 expression in LV-ECS. During the valve maturation stage, LV-ECs orient perpendicular to the flow and migrate into the vessel lumen. Then, LV-ECs elongate to form a bi-layered leaflet with a thick extracellular matrix (ECM). YAP/TAZ activities are gradually increased during the maturation stage, thereby indicating that Hippo YAP/TAZ signaling pathway is regulated by a multitude of signaling mechanisms for LV development. with the formation of two distinct cell populations. LECs from the lymph sacs and LVV-forming endothelial cells (LVV-ECs) from the veins interact to build the LVVs. LVV-ECs quickly aggregate again and invaginate into the vein to create valve leaflets around E12.5. Then, LVVs experience gradual maturation by assembling mural cells to the gap between the LVV-ECs between E14.5 to E16.5. The expression of PROX1, GATA2, and FOXC2 are increased in LVV-ECs and strong expression of VEGFR3 is remained in the LECs that create LVVs as well (128). YAP/TAZ and CTGF are almost absent in LVV-ECs between E12.0 to E14.5 but enriched around E16.5 (44). Consistent with the expression data, Lyve1-Cre; Yap ${ }^{\mathrm{f} / \mathrm{f}}$; $\mathrm{Taz}^{\mathrm{ftf}}$ embryos lack any obvious morphologic defects at E14.5 in LVVs. However, mutants with the complete absence of LVVs at E17.5 indicate that YAP/TAZ activity progressively augments during LVV maturation and YAP/TAZ are necessary for preserving LVV-ECs. We also found that YAP/TAZ positively control PROX1 expression in LVV-ECs (44).

Skin and mesentery lymphatic valves (LVs) start developing around E15.5-E16.5 (18, 130). Differentiation of PROX1 $1^{\text {high }}$, FOXC2 ${ }^{\text {high }}$, and GATA2 $2^{\text {high }}$ LV-ECs is the opening stage of LV development (Fig. 3). OSS generated by lymph flow is one of the most critical factors for FOXC2 and GATA2 expression (14, 131) along with activation of NFATC1 (14) and Wnt/ $\beta$-catenin signaling $(12,100)$. The highly elongated LV-ECs line up along the wall of lymphatic vessels at E16.5, and then they aligned perpendicular to lymph flow at E17.5. ECM molecules such as collagen IV, laminin- $\alpha 5$, fibronectin (FN)-EIIIA, and EMILIN1 are accumulated in between the LV-EC layers around E17.5 $(14,18,130,132)$. Next, the LV-EC layers stretch along the direction of the lymph flow to produce mature LV leaflets after E18.5 $(129,133)$. At E16.5, TAZ is mainly localized in the cytoplasm (45) in LV-Ecs; however, at the maturation stage, TAZ appears to be located in the nucleus $(44,46)$. E18.5 of Lyve1-Cre; Yap ${ }^{\mathrm{f} / \mathrm{f}} ;$ Taz $^{\mathrm{f} / \mathrm{f}}$ embryos show dilated lymphatic vessel with no LVs and immature phenotype with strong expression of LYVE1, VEGFR3, and PROX1 (44). While deletion of YAP/TAZ after birth using tamoxifen delivery system from P1 to P7 leads to a reduction in the LV number with high expression of FOXC2 and PROX1, hyperactivation of YAP/TAZ causes a decrease in LV number with low expression of PROX1 and Integrin- $\alpha 9$ (45). Therefore, a balance in YAP/TAZ activity is important for maintaining the lymphatic valve.

\section{CONCLUDING REMARKS}

In summary, although our understanding of lymphatic vessel functions under physiological or pathological conditions has improved in the past decade, many questions remain unclear. Therefore, it has been considered that the identification of lymphangiogenic modulators and a clear understanding of the involved signaling pathways will provide opportunities to develop therapeutic targets for lymphatic diseases. Among the factors, PROX1 and VEGF-C/VEGFR3 signaling are the most critical regulators of lymphatic vascular development. The Hippo-YAP/TAZ signaling pathway has been established as a key mechanism of regulation of organ size and tissue homeostasis. Recent studies reveal that YAP/TAZ are the vital molecules of the PROX1/VEGFR3 feedback loop in LEC specification, migration, and LV maturation. Based on the current Hippo signaling pathway, it has been hypothesized that MAP4K family kinases acting in parallel to the MST1/2-SAV1 complex can phosphorylate LATS1/2 to inactivate YAP/TAZ in a contextdependent manner. We generated SAV1 conditional knockout mice using two different Cre lines (Lyve1-Cre;Sav ${ }^{\mathrm{ft/f}}$ and Tie2Cre;Sav $\left.1^{\mathrm{f} / \mathrm{f}}\right)$; the mutants showed no obvious phenotypes in the lymphatic vasculature. Interestingly, Czech and colleagues showed that mice constitutively lacking Map4k4 displayed LVs defect and lymphatic flow disorder with increased Prox 1 expression (134). Besides VEGF-C and mechanical stress such as EMC 
stiffness and shear force, multiple signaling pathways are able to control the development of lymphatic vasculature. It must be elucidated whether YAP/TAZ could be regulated by these signaling pathways in a functionally related manner. Moreover, Hippo pathway molecules have been considered as therapeutic targets in several human diseases. Therefore, it is hypothesized that targeting those molecules will provide new therapeutic strategies to cure lymphatic disease in the future.

\section{ACKNOWLEDGEMENTS}

We sincerely apologize as we are unable to cite multiple key research papers due to space limitations. We thank Dr. R. Sathish Srinivasan for his insightful comments. This work is supported by the grant from the National Research Foundation of Korea (2020R1F1A1060680) to B. Cha (2020R1C1C100705011), S. Moon, and (2021R1A2C4001704) W. Kim.

\section{CONFLICTS OF INTEREST}

The authors have no conflicting interests.

\section{REFERENCES}

1. Adams RH and Alitalo K (2007) Molecular regulation of angiogenesis and lymphangiogenesis. Nat Rev Mol Cell Biol 8, 464-478

2. Oliver G and Detmar M (2002) The rediscovery of the lymphatic system: old and new insights into the development and biological function of the lymphatic vasculature. Genes Dev 16, 773-783

3. Harvey NL, Srinivasan RS, Dillard ME et al (2005) Lymphatic vascular defects promoted by Prox1 haploinsufficiency cause adult-onset obesity. Nat Genet 37, 1072-1081

4. Dieterich LC, Seidel CD and Detmar M (2014) Lymphatic vessels: new targets for the treatment of inflammatory diseases. Angiogenesis 17, 359-371

5. Lim K-C, Hosoya T, Brandt W et al (2012) Conditional Gata2 inactivation results in HSC loss and lymphatic mispatterning. J Clin Invest 122, 3705-3717

6. Martel C, Li W, Fulp B et al (2013) Lymphatic vasculature mediates macrophage reverse cholesterol transport in mice. J Clin Invest 123, 1571-1579

7. Randolph GJ, Angeli V and Swartz MA (2005) Dendriticcell trafficking to lymph nodes through lymphatic vessels. Nat Rev Immunol 5, 617-628

8. Da Mesquita S, Louveau A, Vaccari A et al (2018) Functional aspects of meningeal lymphatics in ageing and Alzheimer's disease. Nature 560, 185-191

9. Wiig $H$, Schröder A, Neuhofer $W$ et al (2013) Immune cells control skin lymphatic electrolyte homeostasis and blood pressure. J Clin Invest 123, 2803-2815

10. Ho YC and Srinivasan RS (2020) Lymphatic vasculature in energy homeostasis and obesity. Front Physiol 11, 3

11. Vaahtomeri K, Karaman S, Mäkinen T and Alitalo K (2017) Lymphangiogenesis guidance by paracrine and pericell- ular factors. Genes Dev 31, 1615-1634

12. Cha B, Geng X, Mahamud MR et al (2016) Mechanotransduction activates canonical Wnt/ $\beta$-catenin signaling to promote lymphatic vascular patterning and the development of lymphatic and lymphovenous valves. Genes Dev 30, 1454-1469

13. Tatin F, Taddei A, Weston A et al (2013) Planar cell polarity protein Celsr1 regulates endothelial adherens junctions and directed cell rearrangements during valve morphogenesis. Dev Cell 26, 31-44

14. Sabine A, Agalarov Y, Hajjami HM-E et al (2012) Mechanotransduction, PROX1, and FOXC2 cooperate to control connexin37 and calcineurin during lymphaticvalve formation. Dev Cell 22, 430-445

15. McLatchie LM, Fraser NJ, Main MJ et al (1998) RAMPs regulate the transport and ligand specificity of the calcitonin-receptor-like receptor. Nature 393, 333-339

16. Murtomaki A, Uh MK, Choi YK et al (2013) Notch1 functions as a negative regulator of lymphatic endothelial cell differentiation in the venous endothelium. Development 140, 2365-2376

17. Dunworth WP, Cardona-Costa J, Bozkulak EC et al (2014) Bone morphogenetic protein 2 signaling negatively modulates lymphatic development in vertebrate embryos. Circ Res 114, 56-66

18. Bazigou E, Xie S, Chen C et al (2009) Integrin-alpha9 is required for fibronectin matrix assembly during lymphatic valve morphogenesis. Dev Cell 17, 175-186

19. Kim W and Jho EH (2018) The history and regulatory mechanism of the Hippo pathway. BMB Rep 51, 106-118

20. Misra JR and Irvine KD (2018) The Hippo signaling network and its biological functions. Annu Rev Genet 52, 65-87

21. Ma S, Meng Z, Chen R and Guan K-L (2019) The Hippo pathway: biology and pathophysiology. Annu Rev Biochem 88, 577-604

22. Zanconato F, Cordenonsi $M$ and Piccolo S (2016) YAP/TAZ at the roots of cancer. Cancer Cell 29, 783-803

23. Totaro A, Panciera T and Piccolo S (2018) YAP/TAZ upstream signals and downstream responses. Nat Cell Biol 20, 888-899

24. Piccolo S, Dupont S and Cordenonsi M (2014) The biology of YAP/TAZ: hippo signaling and beyond. Physiol Rev 94, 1287-1312

25. Meng Z, Moroishi T and Guan KL (2016) Mechanisms of Hippo pathway regulation. Genes Dev 30, 1-17

26. Yu FX, Zhao B and Guan KL (2015) Hippo pathway in organ size control, tissue homeostasis, and cancer. Cell $163,811-828$

27. Justice RW, Woods ODF, Nol M and Bryant PJ (1995) The Drosophila tumor suppressor gene warts encodes a homolog of human myotonic dystrophy kinase and is required for the control of cell shape and proliferation. Genes Dev 9, 534-546

28. Xu T, Wang W, Zhang S, Stewart RA and Yu W (1995) Identifying tumor suppressors in genetic mosaics: the Drosophila lats gene encodes a putative protein kinase. Development 121, 1053-1063

29. Tapon N, Harvey KF, Bell DW et al (2002) Salvador promotes both cell cycle exit and apoptosis in Drosophila 
and is mutated in human cancer cell lines. Cell 110, 467-478

30. Harvey KF, Pfleger CM and Hariharan IK (2003) The Drosophila Mst ortholog, hippo, restricts growth and cell proliferation and promotes apoptosis. Cell 114, 457-467

31. Udan RS, Kango-Singh M, Nolo R, Tao C and Halder G (2003) Hippo promotes proliferation arrest and apoptosis in the Salvador/Warts pathway. Nat Cell Biol 5, 914-920

32. Wu S, Huang J, Dong J and Pan D (2003) Hippo encodes a Ste-20 family protein kinase that restricts cell proliferation and promotes apoptosis in conjunction with salvador and warts. Cell 114, 445-456

33. Zheng Y and Pan D (2019) The Hippo signaling pathway in development and disease. Dev Cell 50, 264-282

34. Yu FX and Guan KL (2013) The Hippo pathway: regulators and regulations. Genes Dev 27, 355-371

35. Zhao B, Li L, Tumaneng K, Wang CY and Guan KL (2010) A coordinated phosphorylation by Lats and CK1 regulates YAP stability through $\mathrm{SCF}($ beta-TRCP). Genes Dev 24, 72-85

36. Zhao B, Wei X, Li W et al (2007) Inactivation of YAP oncoprotein by the Hippo pathway is involved in cell contact inhibition and tissue growth control. Genes Dev $21,2747-2761$

37. Huang W, Lv X, Liu C et al (2012) The N-terminal phosphodegron targets TAZ/WWTR1 protein for SCFbetaTrCP-dependent degradation in response to phosphatidylinositol 3-kinase inhibition. J Biol Chem 287, 2624526253

38. Zhang L, Ren F, Zhang Q, Chen Y, Wang B and Jiang J (2008) The TEAD/TEF family of transcription factor Scalloped mediates Hippo signaling in organ size control. Dev Cell $14,377-387$

39. Wu S, Liu Y, Zheng Y, Dong J and Pan D (2008) The TEAD/ TEF family protein Scalloped mediates transcriptional output of the Hippo growth-regulatory pathway. Dev Cell 14, 388-398

40. Kim J, Kim YH, Kim J et al (2017) YAP/TAZ regulates sprouting angiogenesis and vascular barrier maturation. J Clin Invest 127, 3441-3461

41. Sakabe M, Fan J, Odaka Y et al (2017) YAP/TAZ-CDC42 signaling regulates vascular tip cell migration. Proc Natl Acad Sci U S A 114, 10918-10923

42. Wang X, Valls AF, Schermann G et al (2017) YAP/TAZ orchestrate VEGF signaling during developmental angiogenesis. Dev Cell 42, 462-478.e7

43. Giampietro C, Disanza A, Bravi L et al (2015) The actin-binding protein EPS8 binds VE-cadherin and modulates YAP localization and signaling. J Cell Biol $211,1177-1192$

44. Cha B, Ho YC, Geng $X$ et al (2020) YAP and TAZ maintain PROX1 expression in the developing lymphatic and lymphovenous valves in response to VEGF-C signaling. Development 147, dev195453

45. Cho H, Kim J, Ahn JH et al (2019) YAP and TAZ negatively regulate Prox 1 during developmental and pathologic lymphangiogenesis. Circ Res 124, 225-242

46. Sabine A, Bovay E, Demir CS et al (2015) FOXC2 and fluid shear stress stabilize postnatal lymphatic vasculature. J Clin Invest 125, 3861-3877
47. Plouffe SW, Lin KC, Moore JL 3rd et al (2018) The Hippo pathway effector proteins YAP and TAZ have both distinct and overlapping functions in the cell. J Biol Chem 293, 11230-11240

48. Sun C, Mello VD, Mohamed A et al (2017) Common and distinctive functions of the Hippo effectors Taz and Yap in skeletal muscle stem cell function. Stem Cells 35, 1958-1972

49. Escobedo $\mathrm{N}$ and Oliver $\mathrm{G}$ (2016) Lymphangiogenesis: origin, specification, and cell fate determination. Annu Rev Cell Dev Biol 32, 677-691

50. Bui K and Hong YK (2020) Ras pathways on Prox1 and lymphangiogenesis: insights for therapeutics. Front Cardiovasc Med 7, 597374

51. Grimm L, Nakajima H, Chaudhury S et al (2019) Yap1 promotes sprouting and proliferation of lymphatic progenitors downstream of Vegfc in the zebrafish trunk. Elife 8, e42881

52. Azad T, Rensburg HJJ, Lightbody ED et al (2018) A LATS biosensor screen identifies VEGFR as a regulator of the Hippo pathway in angiogenesis. Nat Commun 9, 1061

53. Yeh YW, Cheng CC, Yang ST et al (2017) Targeting the VEGF-C/VEGFR3 axis suppresses Slug-mediated cancer metastasis and stemness via inhibition of KRAS/YAP1 signaling. Oncotarget 8, 5603-5618

54. Hamaratoglu F, Willecke M, Kango-Singh M et al (2006) The tumour-suppressor genes NF2/Merlin and expanded act through Hippo signalling to regulate cell proliferation and apoptosis. Nat Cell Biol 8, 27-36

55. Willecke M, Hamaratoglu F, Kango-Singh M et al (2006) The fat cadherin acts through the hippo tumor-suppressor pathway to regulate tissue size. Curr Biol 16, 2090-2100

56. Kuta A, Mao Y, Martin T et al (2016) Fat4-Dchs1 signalling controls cell proliferation in developing vertebrae. Development 143, 2367-2375

57. Alders M, Al-Gazali L, Cordeiro I et al (2014) Hennekam syndrome can be caused by FAT4 mutations and be allelic to Van Maldergem syndrome. Hum Genet 133, 1161-1167

58. Betterman KL, Sutton DL, Secker GA et al (2020) Atypical cadherin FAT4 orchestrates lymphatic endothelial cell polarity in response to flow. J Clin Invest 130, 3315-3328

59. Cheong SS, Akram KM, Matellan C et al (2020) The planar polarity component VANGL2 is a key regulator of mechanosignaling. Front Cell Dev Biol 8, 577201

60. Choi HJ, Zhang H, Park H et al (2015) Yes-associated protein regulates endothelial cell contact-mediated expression of angiopoietin-2. Nat Commun 6, 6943

61. Hägerling R, Hoppe E, Dierkes C et al (2018) Distinct roles of VE-cadherin for development and maintenance of specific lymph vessel beds. EMBO J 37, e98271

62. Yang Y, Cha B, Motawe ZY, Srinivasan RS, Scallan JP (2019) VE-Cadherin is required for lymphatic valve formation and maintenance. Cell Rep 28, 2397-2412.e4

63. Au AC, Hernandez PA, Lieber E et al (2010) Protein tyrosine phosphatase PTPN14 is a regulator of lymphatic function and choanal development in humans. Am J Hum Genet 87, 436-444

64. Liu X, Yang N, Figel SA et al (2013) PTPN14 interacts 
with and negatively regulates the oncogenic function of YAP. Oncogene 32, 1266-1273

65. Wilson KE, Li YW, Yang N, Shen $\mathrm{H}$, Orillion AR and Zhang J (2014) PTPN14 forms a complex with Kibra and LATS1 proteins and negatively regulates the YAP oncogenic function. J Biol Chem 289, 23693-23700

66. Wang W, Huang J, Wang X et al (2012) PTPN14 is required for the density-dependent control of YAP1. Genes Dev 26, 1959-1971

67. Dupont S, Morsut L, Aragona M et al (2011) Role of YAP/TAZ in mechanotransduction. Nature 474, 179-183

68. Panciera T, Azzolin L, Cordenonsi $M$ et al (2017) Mechanobiology of YAP and TAZ in physiology and disease. Nat Rev Mol Cell Biol 18, 758-770

69. Wang KC, Yeh YT, Nguyen P et al (2016) Flow-dependent YAP/TAZ activities regulate endothelial phenotypes and atherosclerosis. Proc Natl Acad Sci U S A 113, 1152511530

70. Wang L, Luo JY, Li B et al (2016) Integrin-YAP/TAZ-JNK cascade mediates atheroprotective effect of unidirectional shear flow. Nature 540, 579-582

71. Nakajima H, Yamamoto K, Agarwala $S$ et al (2017) Flow-dependent endothelial YAP regulation contributes to vessel maintenance. Dev Cell 40, 523-536.e6

72. Nakajima $\mathrm{H}$ and Mochizuki N (2017) Flow patterndependent endothelial cell responses through transcriptional regulation. Cell Cycle 16, 1893-1901

73. Choi D, Park E, Jung E et al (2017) Laminar flow downregulates Notch activity to promote lymphatic sprouting. J Clin Invest 127, 1225-1240

74. Geng X, Yanagida K, Akwii RG et al (2020) S1PR1 regulates the quiescence of lymphatic vessels by inhibiting laminar shear stress-dependent VEGF-C signaling. JCI Insight 5, e137652

75. Liu Z, Wei Y, Zhang L et al (2019) Induction of store-operated calcium entry (SOCE) suppresses glioblastoma growth by inhibiting the Hippo pathway transcriptional coactivators YAP/TAZ. Oncogene 38, 120-139

76. Choi D, Park E, Jung E et al (2019) Piezo1 incorporates mechanical force signals into the genetic program that governs lymphatic valve development and maintenance. JCl Insight 4, e125068

77. Nonomura K, Lukacs V, Sweet DT et al (2018) Mechanically activated ion channel PIEZO1 is required for lymphatic valve formation. Proc Natl Acad Sci U S A $115,12817-12822$

78. Pathak MM, Nourse JL, Tran T et al (2014) Stretchactivated ion channel Piezo1 directs lineage choice in human neural stem cells. Proc Natl Acad Sci U S A 111, 16148-16153

79. Zhou T, Gao B, Fan Y et al (2020) Piezo1/2 mediate mechanotransduction essential for bone formation through concerted activation of NFAT-YAP1- $\beta$-catenin. Elife 9, e52779

80. Wang L, You X, Lotinun S, Zhang L, Wu N and Zou W (2020) Mechanical sensing protein PIEZO1 regulates bone homeostasis via osteoblast-osteoclast crosstalk. Nat Commun 11, 282

81. Sun Z, Guo SS and Fässler R (2016) Integrin-mediated mechanotransduction. J Cell Biol 215, 445-456

82. Rausch $\vee$ and Hansen CG (2020) The Hippo pathway,
YAP/TAZ, and the plasma membrane. Trends Cell Biol $30,32-48$

83. Frye M, Taddei A, Dierkes C et al (2018) Matrix stiffness controls lymphatic vessel formation through regulation of a GATA2-dependent transcriptional program. Nat Commun 9, 1511

84. Aragona M, Panciera T, Manfrin A et al (2013) A mechanical checkpoint controls multicellular growth through YAP/TAZ regulation by actin-processing factors. Cell 154, 1047-1059

85. Planas-Paz L, Strilić B, Goedecke A, Breier G, Fässler R and Lammert E (2012) Mechanoinduction of lymph vessel expansion. EMBO J 31, 788-804

86. Yu FX, Zhao B, Panupinthu N et al (2012) Regulation of the Hippo-YAP pathway by G-protein-coupled receptor signaling. Cell 150, 780-791

87. Hoopes SL, Willcockson HH and and Caron KM (2008) Characteristics of multi-organ lymphangiectasia resulting from temporal deletion of calcitonin receptor-like receptor in adult mice. PLoS One 7, e45261

88. Lee SJ, Chan TH, Chen TC, Liao BK, Hwang PP and Lee $\mathrm{H}$ (2008) LPA1 is essential for lymphatic vessel development in zebrafish. FASEB J 22, 3706-3715

89. Sumida H, Noguchi K, Kihara Y et al (2010) LPA4 regulates blood and lymphatic vessel formation during mouse embryogenesis. Blood 116, 5060-5070

90. Lin $\mathrm{Cl}$, Chen CN, Huang MT et al (2008) Lysophosphatidic acid up-regulates vascular endothelial growth factor-C and lymphatic marker expressions in human endothelial cells. Cell Mol Life Sci 65, 2740-2751

91. Yoon CM, Hong BS, Moon HG et al (2008) Sphingosine1-phosphate promotes lymphangiogenesis by stimulating S1P1/Gi/PLC/Ca2 + signaling pathways. Blood 112, 11291138

92. Kim W, Kim M and Jho EH (2013) Wnt/beta-catenin signalling: from plasma membrane to nucleus. Biochem J 450, 9-21

93. Nusse R and Clevers H (2017) Wnt/beta-catenin signaling, disease, and emerging therapeutic modalities. Cell 169 985-999

94. Clevers H and Nusse R (2012) Wnt/beta-catenin signaling and disease. Cell 149, 1192-1205

95. Kim M and Jho EH (2014) Cross-talk between Wnt/betacatenin and Hippo signaling pathways: a brief review. BMB Rep 47, 540-545

96. Azzolin L, Zanconato F, Bresolin S et al (2012) Role of TAZ as mediator of Wnt signaling. Cell 151, 1443-1456

97. Azzolin L, Panciera T, Soligo S et al (2014) YAP/TAZ incorporation in the beta-catenin destruction complex orchestrates the Wnt response. Cell 158, 157-170

98. Park HW, Kim YC, Yu B et al (2015) Alternative Wnt signaling activates YAP/TAZ. Cell 162, 780-794

99. Lutze G, Haarmann A, Toukam JAD, Buttler K, Wilting J and Becker J (2019) Non-canonical WNT-signaling controls differentiation of lymphatics and extension lymphangiogenesis via RAC and JNK signaling. Sci Rep 9, 4739

100. Cha B, Geng X, Mahamud MR et al (2018) Complementary Wnt sources regulate lymphatic vascular development via PROX1-dependent $\mathrm{Wnt} / \beta$-catenin signaling. Cell Rep 25, 571-584.e5 
101. Majumder S, Crabtree JS, Golde TE, Minter LM, Osborne BA and Miele L (2021) Targeting Notch in oncology: the path forward. Nat Rev Drug Discov 20, 125-144

102. Bray SJ (2016) Notch signalling in context. Nat Rev Mol Cell Biol 17, 722-735

103. Tschaharganeh DF, Chen X, Latzko P et al (2013) Yesassociated protein up-regulates Jagged-1 and activates the Notch pathway in human hepatocellular carcinoma. Gastroenterology 144, 1530-1542.e12

104. Kim W, Khan SK, Gvozdenovic-Jeremic J et al (2017) Hippo signaling interactions with $W n t / \beta$-catenin and Notch signaling repress liver tumorigenesis. J Clin Invest 127, 137-152

105. Kang J, Yoo J, Lee S et al (2010) An exquisite crosscontrol mechanism among endothelial cell fate regulators directs the plasticity and heterogeneity of lymphatic endothelial cells. Blood 116, 140-150

106. Fatima A, Culver A, Culver F et al (2014) Murine Notch1 is required for lymphatic vascular morphogenesis during development. Dev Dyn 243, 957-964

107. Niessen K, Zhang G, Ridgway JB et al (2011) The Notch1-Dll4 signaling pathway regulates mouse postnatal lymphatic development. Blood 118, 1989-1997

108. Srinivasan RS, Dillard ME, Lagutin OV et al (2007) Lineage tracing demonstrates the venous origin of the mammalian lymphatic vasculature. Genes Dev 21, 24222432

109. Wigle JT and Oliver G (1999) Prox1 function is required for the development of the murine lymphatic system. Cell 98, 769-778

110. Yaniv K, Isogai S, Castranova D, Dye L, Hitomi J and Weinstein BM (2006) Live imaging of lymphatic development in the zebrafish. Nat Med 12, 711-716

111. Srinivasan RS, Geng X, Yang Y et al (2010) The nuclear hormone receptor Coup-TFII is required for the initiation and early maintenance of Prox 1 expression in lymphatic endothelial cells. Genes Dev 24, 696-707

112. Hong $\mathrm{YK}$, Harvey N, Noh $\mathrm{YH}$ et al (2002) Prox 1 is a master control gene in the program specifying lymphatic endothelial cell fate. Dev Dyn 225, 351-357

113. François M, Caprini A, Hosking B et al (2008) Sox18 induces development of the lymphatic vasculature in mice. Nature 456, 643-647

114. Srinivasan RS, Escobedo N, Yang Y et al (2014) The Prox1-Vegfr3 feedback loop maintains the identity and the number of lymphatic endothelial cell progenitors. Genes Dev 28, 2175-2187

115. Koltowska K, Lagendijk AK, Pichol-Thievend $\mathrm{C}$ et al (2015) Vegfc regulates bipotential precursor division and Prox 1 expression to promote lymphatic identity in zebrafish. Cell Rep 13, 1828-1841

116. Yang $Y$, García-Verdugo JM, Soriano-Navarro $M$ et al (2012) Lymphatic endothelial progenitors bud from the cardinal vein and intersomitic vessels in mammalian embryos. Blood 120, 2340-2348

117. Hägerling R, Pollmann C, Andreas $M$ et al (2013) A novel multistep mechanism for initial lymphangiogenesis in mouse embryos based on ultramicroscopy. EMBO J $32,629-644$

118. Karkkainen MJ, Haiko P, Sainio K et al (2004) Vascular endothelial growth factor $\mathrm{C}$ is required for sprouting of the first lymphatic vessels from embryonic veins. Nat Immunol 5, 74-80

119. Semo J, Nicenboim J and Yaniv K (2016) Development of the lymphatic system: new questions and paradigms. Development 143, 924-935

120. Chen H, Griffin C, Xia L and Srinivasan RS (2014) Molecular and cellular mechanisms of lymphatic vascular maturation. Microvasc Res 96, 16-22

121. Martinez-Corral I, Ulvmar MH, Stanczuk L et al (2015) Nonvenous origin of dermal lymphatic vasculature. Circ Res 116, 1649-1654

122. James JM, Nalbandian A and Mukouyama YS (2013) TGF $\beta$ signaling is required for sprouting lymphangiogenesis during lymphatic network development in the skin. Development 140, 3903-3914

123. Petrova TV, Karpanen T, Norrmén C et al (2004) Defective valves and abnormal mural cell recruitment underlie lymphatic vascular failure in lymphedema distichiasis. Nat Med 10, 974-981

124. Yuan L, Moyon D, Pardanaud L et al (2002) Abnormal lymphatic vessel development in neuropilin 2 mutant mice. Development 129, 4797-4806

125. Mahamud MR, Geng X, Ho YC et al (2019) GATA2 controls lymphatic endothelial cell junctional integrity and lymphovenous valve morphogenesis through miR126. Development 146, dev184218

126. Srinivasan RS and Oliver G (2011) Prox1 dosage controls the number of lymphatic endothelial cell progenitors and the formation of the lymphovenous valves. Genes Dev 25, 2187-2197

127. Tammela T and Alitalo K (2010) Lymphangiogenesis: Molecular mechanisms and future promise. Cell 140, 460-476

128. Geng X, Cha B, Mahamud MR et al (2016) Multiple mouse models of primary lymphedema exhibit distinct defects in lymphovenous valve development. Dev Biol 409, 218-233

129. Geng X, Cha B, Mahamud MR and Srinivasan RS (2017) Intraluminal valves: development, function and disease. Dis Model Mech 10, 1273-1287

130. Norrmén C, Ivanov Kl, Cheng J et al (2009) FOXC2 controls formation and maturation of lymphatic collecting vessels through cooperation with NFATc1. J Cell Biol 185, 439-457

131. Kazenwadel J, Betterman KL, Chong CE et al (2015) GATA2 is required for lymphatic vessel valve development and maintenance. J Clin Invest 125, 2979-2994

132. Danussi C, Belluz LDB, Pivetta E et al (2013) EMILIN1/ $\alpha 9 \beta 1$ integrin interaction is crucial in lymphatic valve formation and maintenance. Mol Cell Biol 33, 4381-4394

133. Bazigou E, Wilson JT and Moore Jr JE (2014) Primary and secondary lymphatic valve development: molecular, functional and mechanical insights. Microvasc Res 96, 38-45

134. Roth Flach RJ, Guo CA, Danai LV et al (2016) Endothelial mitogen-activated protein kinase kinase kinase kinase 4 is critical for lymphatic vascular development and function. Mol Cell Biol 36, 1740-1749 